\title{
Environmental Pressure Problem Identified
}

National Cancer Institute

\section{Source}

National Cancer Institute. Environmental Pressure Problem Identified. NCI Thesaurus.

Code C139493.

Device performance was affected by the pressure, or changes in pressure, of the environment in which it was used. 